\title{
The transition to turbulence of the torsional Couette flow
}

\author{
A. Cros and P. Le Gal \\ Institut de Recherche sur les Phénomènes Hors Equilibre, \\ 49, rue F. Joliot-Curie, 13384, Marseille, cédex 13, France \\ e-mail: legal@irphe.univ-mrs.fr - Web page: http://www.irphe.univ-mrs.fr
}

\begin{abstract}
This work is devoted to the experimental study of the transition to turbulence of a flow confined in a narrow gap between a rotating and a stationary disk. The experimental device, presented in Figure 1, consists of a water-filled cylindrical casing in which the rotating disk is immersed. The top lid of the container plays the role of the stationary disk. The radius of the stainless steel disk is $140 \mathrm{~mm}$ and its thickness is $13 \mathrm{~mm}$. It is painted in black to enhance visualization which is realized with kalliroscope flakes.
\end{abstract}

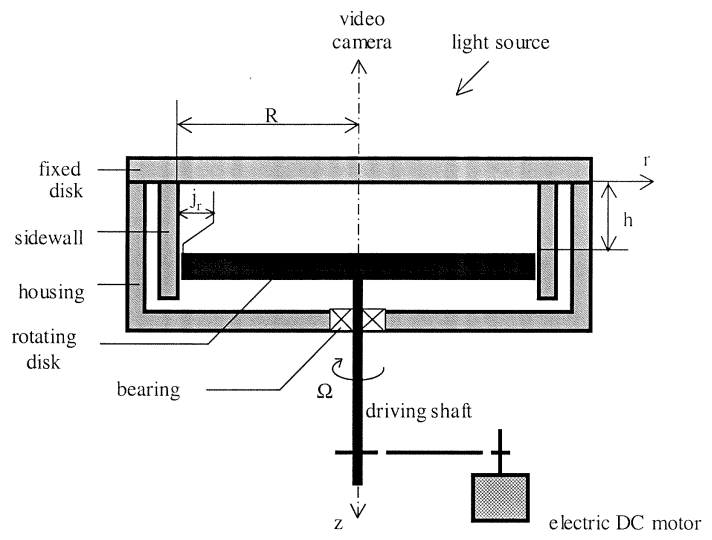

Figure 1: Experimental setup.

The drive shaft goes through the bottom of the tank and is connected to a d.c. electric motor whose rotating velocity can be varied from $\Omega=0$ to $200 \mathrm{rpm}$ with an accuracy better than $0.2 \%$. The stationary disk is a $20 \mathrm{~mm}$ thick plexiglass plate, so that the flow can be observed through it. The distance between the rotating disk and the fixed one is set to $2 \mathrm{~mm}$. A CCD video camera is placed on the rotation axis and can rotate if necessary with a velocity which can be adjusted in order to observe the waves in their rotating frame. This video camera is finally connected to a computer, and images can be captured in real time.

In the case of interest here, as the fluid layer thickness is of the same order of magnitude than the boundary layer depths, the azimuthal velocity axial gradient is nearly constant and this rotating disk flow tends to be a torsional Couette flow (see Cros et al., 2002). As in the plane Couette flow or the cylindrical Couette flow, transition to turbulence occurs via the appearance of turbulent domains inside a laminar background. Nevertheless, we show that, in the rotating disk case, the nucleation of turbulent 

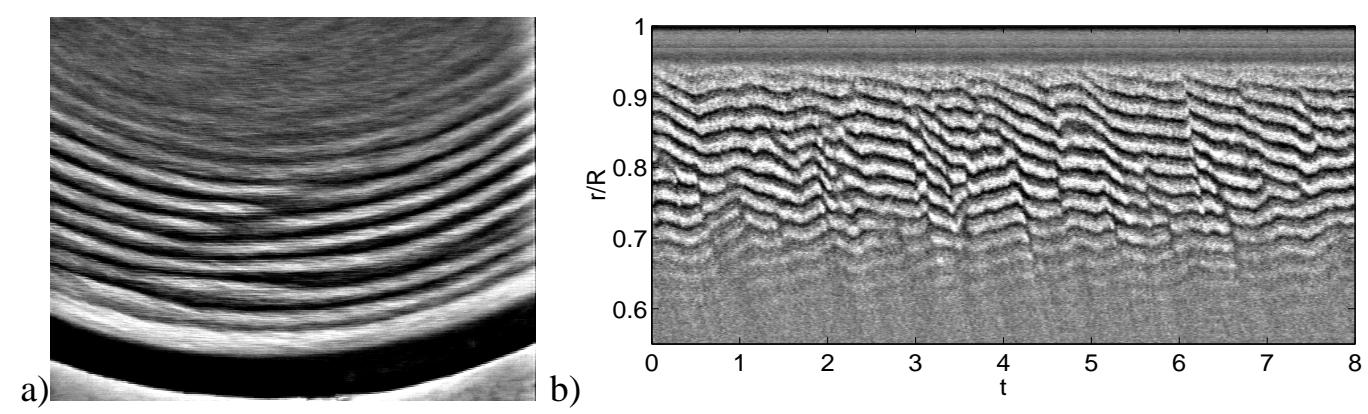

Figure 2: a) Defect in the spiral roll pattern. b) Space-time diagram along a radius

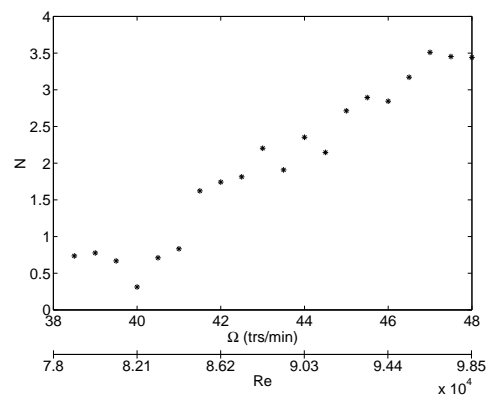

Figure 3: Evolution of the total number of defects versus Reynolds number.

spirals, previously called "solitary waves" in the rotating disk flow literature, is connected to the birth of structural defects in a periodic underlying spiral roll pattern.

Figure 2 presents such a defect and a spatio-temporal diagram realized along the radial direction which exhibits this defect-turbulence (Coullet et al. 1989). We then performed a statistical study of these defects and showed that their occurrence obeys to a Poisson law near their threhold. We observe in particular that their number increases similarly to what is proposed by theoretical studies (Shraiman et al., 1992). Our results are also in agreement with the interpretation of these defects as homoclinic orbits of a dynamical system nearby a saddle-node critical point (Afraimovich et al., 1995). Figure 3 shows the evolution of the total number of these defects as a function of the Reynolds number.
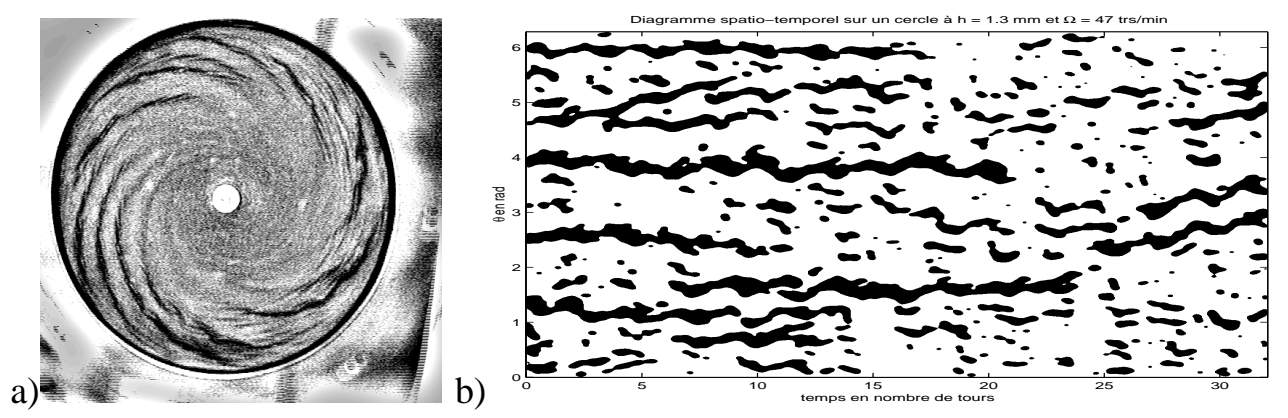

Figure 4: a) Visualization of the turbulent spirals. b) Space-time diagram (along a circle) showing the turbulent spirals (in black) inside the laminar flow (in white) 
As the rotation rate is increased, the time duration separating two consecutive defects decreases dramaticaly and the defects are then associated to strong amplitude modulations. A similarity with the "MAWs" of Brusch et al. (2000) is striking. In fact, these modulations act as seeds for the turbulent spirals visualized in Figure 4.

Very near their observation threshold, these turbulent structures that, we believe to be the equivalent of the turbulent spirals of the cylindrical Couette flow (note that although their traditionnal appellation, they are not spirals in this case but rather helices!) have a very short life time. As the rotation rate is still increased, this lifetime increases until a threshold is reached where they then finally form permanent turbulent spirals arranged nearly periodically all around a circumference. However, since the number of these turbulent spirals decreases with the rotational frequency, the transition to a fully turbulent regime is not achieved. Thus the turbulent fraction of the pattern saturates to a value close to 0.5. Figure 5 presents the evolution of this turbulent fraction with the Reynolds number. As it can be seen, this turbulent fraction presents a power law critical behaviour near threshold with an exponent $\beta=0.3$.

a)

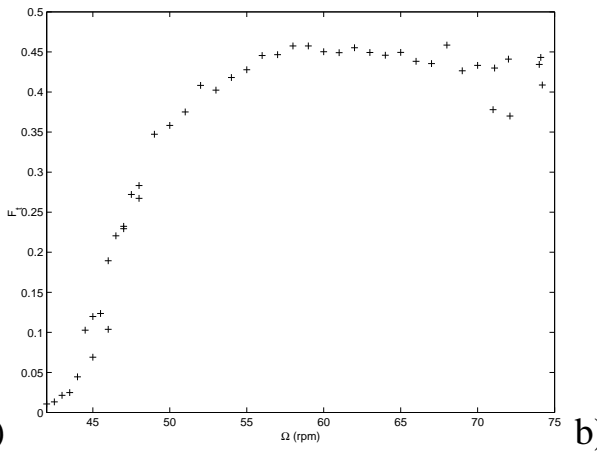

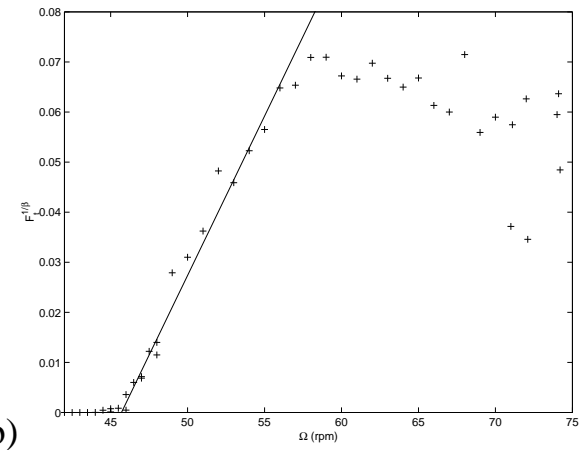

Figure 5: a) Evolution of the turbulent fraction versus Reynolds number. b) At threshold, an exponent $\beta=0.3 \pm 0.01$ can be determined.

Another exponent of the transition has also been measured. As presented in Figure 6, the statistics of the length of the laminar domains follows an exponential decrease which permits to define a characteristic length. The evolution of these laminar domains lengths versus the Reynolds number presents a divergence with a power law with an exponent $\alpha=-\frac{1}{2}$. Therefore, although the transition is not completed, it seems that it shares some features with the Space-Time Intermittency scenario (Chaté, 1987).

Therefore, although it seems that a universal behavior is still lacking for this type of transition to turbulence, we note that both the values of $\alpha$ and $\beta$ agree with the ones measured by Daviaud et al. (1990) in their Rayleigh-Bénard convection experiment. Moreover, the saturation of the turbulent fraction near 0.5 is reminiscent of the amazing periodization of turbulence discovered by Prigent et al. (2002) in the plane and in the cylindrical Couette flow. 

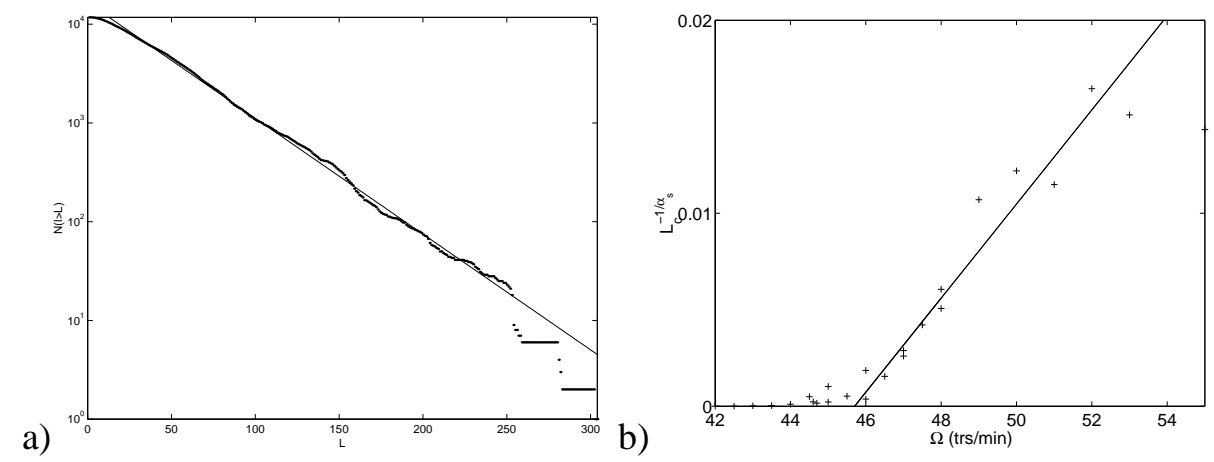

Figure 6: a) Exponential behaviour of the histogram of the laminar lengths. $b$ ) Measure of the exponent $\alpha=-\frac{1}{2}$ of the critical behaviour of the laminar length.

\section{REFERENCES}

[1] Cros A. and Le Gal P., Spatio-temporal intermittency in the torsional Couette flow between a rotating and a stationary disk, Phys. Fluids, 14(11),3755, 2002.

[2] Coullet P., Gil L. and Lega J., Defect-mediated turbulence, Phys. Rev. Lett. 62(14), 1619, 1989.

[3] Shraiman B.I., Pumir A., Van Saarloos W., Hohenberg P.C, Chaté H. and Holen M., Spatiotemporal chaos in the one-dimensional complex Ginzburg-Landau equation, Physica D, 57, $241,1992$.

[4] Afraimoich V.S; and Bunimovich L.A., Density of defects and spatial entropy in extended systems, Physica D 80, 277, 1995.

[5] Brusch L., Zimmerman M.G., van Hecke M., Bär M. and Torcini A., Modulated amplitude waves and the transition from phase to defect chaos. Phys. Rev. Lett. 85 (1), 86, 2000.

[6] Chaté H. and Manneville P., Transition to turbulence via spatio-temporal intermittency, Phys. Rev. E, 58, 112, 1987.

[7] Daviaud F., Bonetti M. and Dubois M., Transition to turbulence via spatio-temporal intermittency in one-dimensional Rayleigh-Bénard convection, Phys. Rev. A, 42, 3388, 1990.

[8] Prigent A., Gregoire G., Chaté H., Dauchot O. and Van Saarloos W., Large-scale finite wavelength modulation within turbulent shear flows. Phys. Rev. Lett 89, 014501, 2002. 